Books, videos, CD-ROMs, DVDs and any other relevant items submitted for a review in the $B D J$ should be addressed to: Kate Maynard, Assistant Editor, British Dental Journal, NPG, 4-6 Crinan Street, London N1 9XW

\section{Adhesive technology for restorative dentistry}

\author{
J-F. Roulet, G. Vanherle \\ UK: Quintessence \\ price $€ 73$, pp 200 \\ ISBN 1850971072
}

Adhesive dentistry is an area of clinical practice where the technology of the adhesives and the techniques that are being developed to use with them are continuously changing. In their preface the editors of this book question the need for a further text in this field but then point out that there is a need for textbooks to bridge the gap between accepted treatment modes and new techniques. They set out to do this with Adhesive technology for restorative dentistry, which is based on the proceedings of the third symposium on adhesive dentistry in 2001.

The book is divided into four sections, the first dealing with aesthetic dentistry, the second with minimally invasive dentistry, the third covering the use of adhesives with ceramics and metals and finally some comments about a continued need for an holistic approach to treating patients.

The first section contains some very useful clinical guidelines including the use of matrices to guide the preparation and restoration of teeth with layered composite resins. This is an area where clinical practice has outstripped clinical education and these clear preparation guides are both timely and useful.

The second section provides a very pertinent overview of cavity design for minimal adhesive restorations, taking the reader from G.V. Black to tunnel preparations. It is interesting to reflect that the principles described by Black are as valid today as they were in the nineteenth century, however modern materials allow us to satisfy these principles with much less destructive preparations in teeth.

The third component of this book sets out the rationale for the use of adhesive
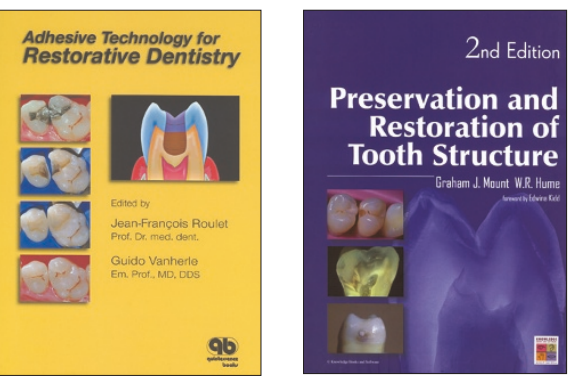

lutes with metals and ceramic restoration, clearly establishing the benefits of resins for reinforcement of all ceramic restorations.

Finally Mjor (the author of the final chapter) and Roulet reflect on the changes in clinical practice that have been achieved with adhesive technology. Mjor in particular places emphasis on the need for realistic appraisal of new techniques in a general practice setting and the changing emphasis of consumer demand with aesthetics becoming a principle concern, displacing restoration longevity as the prime mover for materials choice.

This book is meticulously illustrated where appropriate and provides a good balance between clinical practice, science and philosophy to inform care for patients using modern adhesive materials. It would prove a valuable reference for all dentists who have an interest in adhesive techniques, both in relation to current technology and with regard to future developments in this expanding field.

\section{A. Walls}

\section{Preservation and restoration of tooth structure}

G.J. Mount, W.R. Hume
Australia: Knowledge Books and Software
price £59.95, pp 365

ISBN 192082474X

This is the second edition of a book originally published in 1998. It has a foreword by Edwina Kidd, whose association must surely endorse it as an important reference text.

Initially, it is difficult to see where this book fits among other texts. It is fairly large and too heavy to be easily portable, and it covers some sub-topics such as tooth development and pulpal dynamics in too superficial a way to replace other books dedicated to these subjects. However, its real strength lies in the integrated approach to the topic addressed by the title, whereby dental disease prevention, reversal and repair is approached in relation to tooth structure, the biology of disease and the physical chemical and biological properties of repair techniques when these are called for. The reader needs to sign up to the whole concept presented, and to do this must read the whole book. Once this is done, it is possible to 'dip in and out' of the various sections with a proper understanding.

Chapter 6 introduces a caries-risk model, called 'the Traffic Light - Matrix model'.

This is designed to "collect data from individual patients and develop personalised treatment solution'. While the full model may be somewhat cumbersome for all applications, its concept is valid and it would be helpful in determining individual recall intervals for patients. There are really good sections on Risk Assessment, Lifestyle Impacts and Remineralisation Aids. They are great food for reflective thought for undergraduates and experienced practitioners alike, and the philosophy is definitely compatible in practice with extended roles for PCDs.

Inevitably, some teeth require operative intervention because the carious process has proceeded too far or because previous restorations have failed. Approximately two thirds of the book is devoted broadly to this aspect. The new method of identification and classification of lesions of the tooth crown, as opposed to G.V. Black's classification, first introduced in the previous edition of this book, makes even more sense in this second edition. The authors state that this is not meant to dictate either cavity design or restorative material but to encourage preservation of tooth structure. It does this by explaining principles of integration of cavity design, choice of restorative material and remineralisation potential.

One or two of the figures do not quite illustrate what they claim, but this is a minor criticism. All in all, this book is thoroughly to be recommended. 